\title{
Origin of the medial ulnar collateral ligament on the pediatric elbow
}

\author{
Michael Zell $\cdot$ Jerry R. Dwek $\cdot$ Eric W. Edmonds
}

Received: 14 May 2013/ Accepted: 20 July 2013/Published online: 10 August 2013

(C) EPOS 2013

\begin{abstract}
Purpose Surgical reconstruction of the adult anterior bundle of the medial ulnar collateral elbow ligament (UCL) is a common and established treatment that yields satisfactory results. Children sustain these injuries less frequently, and surgical intervention is complicated by the juxtaposed medial epicondyle apophysis. The purpose of this study was to identify the anatomical origin of the pediatric UCL and determine if this location changes with elbow maturity.

Methods A retrospective analysis of children with an elbow MRI between 2009 and 2012 was performed. Ninety children (68 boys, 22 girls), mean age 12.8 years (range $6-18)$, were grouped by age $(<11,11-13$, and $>13)$ and gender. Measurements of UCL width and UCL midpoint distance from medial epicondyle apophysis were recorded on coronal T1 images utilizing digital PACS software.

Results Across all groups, boys had a wider UCL than girls $(4.05 \pm 0.16 \mathrm{~mm}$ vs $3.72 \pm 0.20 \mathrm{~mm}, p=0.03)$;
\end{abstract}

M. Zell · E. W. Edmonds

Department of Orthopedic Surgery, University of California

San Diego, San Diego, CA 92123, USA

J. R. Dwek

Department of Radiology, Rady Children's Hospital and Health Center, San Diego, CA, USA

J. R. Dwek

Department of Radiology, University of California San Diego,

San Diego, CA, USA

E. W. Edmonds $(\bowtie)$

Department of Orthopedic Surgery, Rady Children's Hospital and Health Center, 3030 Children's Way, Suite 410, San Diego, CA 92123, USA

e-mail: ewedmonds@rchsd.org however, there was no difference in the anatomical origin of the UCL relative to the medial epicondyle apophysis between gender $(p=0.52)$, between gender age-matched groups, or within gender age-matched groups. Yet, the anatomic origin of the UCL always remained medial to the cartilaginous interface of the apophysis with the osseous distal humerus and was centered approximately $3 \mathrm{~mm}$ medial to the lateral edge of the apophysis.

Conclusion Regardless of age or gender, the humeral origin for the medial ulnar collateral ligament is medial to the interface between the medial epicondyle apophysis and distal humerus, which has surgical implications for anatomic reconstruction in children.

Keywords Children - Medial epicondyle apophysis . Ulnar collateral ligament $\cdot$ Reconstruction

\section{Introduction}

The medial elbow ulnar collateral ligament (UCL) complex is comprised of three bundles: anterior, transverse, and posterior [1]. The anterior bundle (aUCL) originates from the medial humeral epicondyle and inserts on the medial aspect of the coronoid process of the ulna at the sublime tubercle, whereas the posterior bundle inserts on the posteromedial olecranon, with the transverse bundle connecting the two insertion points on the ulna (Fig. 1). The anterior bundle has the greatest biomechanical significance, serving as the primary stabilizer of the elbow against valgus stress during functional range of motion with elbow flexion between $20^{\circ}$ and $120^{\circ}$ [2]. Overhead athletes (such as those participating in baseball, volleyball, tennis, gymnastics, wrestling, and water polo) are particularly vulnerable to injury of this ligament due to the excessive valgus 


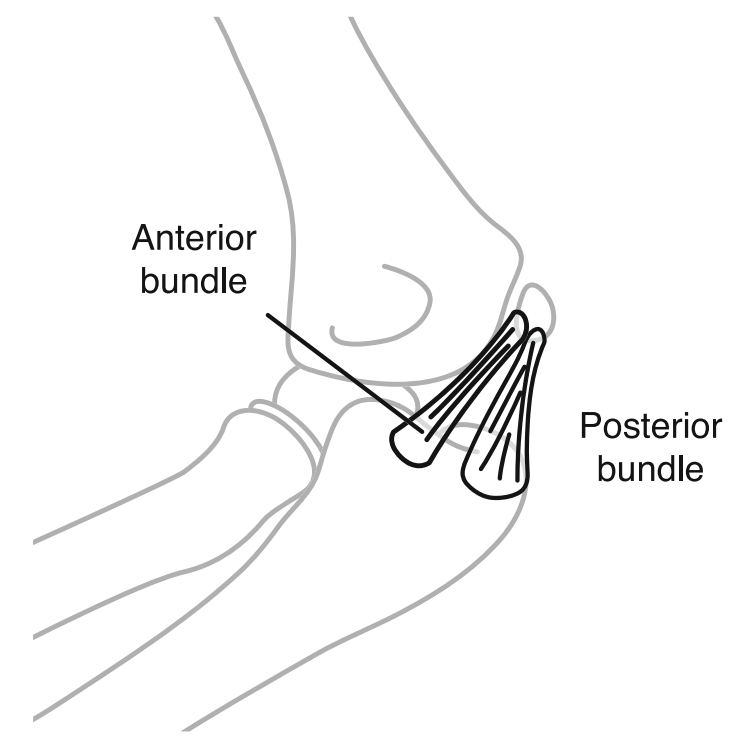

Fig. 1 Line drawing of a 7-year-old elbow demonstrating the origin and insertion of the anterior and posterior bundles of the ulnar collateral ligament relative to the medial epicondyle apophysis

force placed on the elbow during play [3]. During the latecocking and early acceleration phases of the overhead throwing or striking motion, the forces on the aUCL approach physiological limits, leading to repetitive microtrauma and occasionally outright failure [3]. Moreover, the aUCL may be torn with acute upper extremity trauma, and is often torn in an acute elbow dislocation. Although this injury is relatively uncommon, it has clearly become a clinically significant problem, as highlighted by recent literature regarding the injury rate and reconstruction of the aUCL in adolescent athletes [2,4]. Finally, if the aUCL is attached primarily to the apophysis of the medial epicondyle there may be stability issues related to isolated medial epicondyle fractures.

Child athletes are predisposed to unique injuries related to growth and development, and are at particular risk of unique chronic repetitive stress injuries related to two specific factors: (1) open growth plates and (2) the evolution of youth sports from seasonal activities to yearround training [2]. During development, elbow growth occurs at three different apophyses and three distinct physes that also serve as attachment points for key elbow stabilizers and are thus vulnerable to injury [5]. Risk factors have been identified that increase injuries to the elbows of young athletes that can be basically defined as overuse [6]. These injuries usually involve the medial aspect of the elbow, and in the young patient can result primarily in either apophysitis or medial epicondyle fracture. Approximately 35 million children and adolescents participate in sports annually in the United States, and more than 3.5 million each year are treated for sportsrelated injuries. In particular, upper extremity and elbow injuries have shown a marked increase in incidence, with $20-40 \%$ of 9- to 12-year-old baseball players and $50-70 \%$ of adolescent players developing elbow pain annually [1]. This trend is also seen in other athletic activities that similarly subject participants to overhead (or throwing) or upper extremity weight-bearing forces, such as gymnastics and football.

Besides direct injury to the aUCL, there may be an indirect injury related to medial epicondyle fractures. Previous authors have suggested that the stability of the elbow may be severely compromised in the setting of medial epicondyle fractures (even if minimally displaced), due to the possibility of traumatic lengthening of the structure of the aUCL at the moment of injury and/or the persistent shortening of the aUCL during the aftermath of these fractures $[7,8]$.

Consequently, the once-rare aUCL injury among the pediatric population is becoming increasingly common. For children, the initial treatment is conservative management: rest, bracing, NSAIDs, and physical therapy with a gradual return to activity. For many young athletes, this approach is sufficient, although a 2001 study found that among young athletes receiving nonoperative treatment for aUCL injuries, only $42 \%$ had returned to their previous level of competition at a mean 24.5 weeks of treatment [9]. Surgical reconstruction of the aUCL is a popular procedure to restore valgus stability to the elbow in adults, and is becoming more popular for the adolescent athlete $[6,10$, 11]. A recent case series demonstrated that 58 of 60 adolescent athletes undergoing this surgery were able to participate in their sport at a level equivalent to or higher than their previous level within 6 months [12]. However, to our knowledge, no study has been performed specifically on a child with a skeletally immature elbow regarding reconstruction of the aUCL. Anatomic reconstruction of the aUCL in the skeletally immature could result in a variety of potential complications, including poor fixation through the apophyseal cartilage, increased risk of medial epicondyle fracture due to disruption of the apophysis, as well as minor growth disturbance of the apophysis.

There has been some previous research utilizing MRI to assess injuries to the pediatric elbow. One study assessing the injuries incurred by "little leaguer elbow" demonstrated that the aUCL inserted close to the apophysis of the medial epicondyle, but this study was limited to nine patients all in the same age group [13]. Another study looking at 20 teenagers also confirmed that the appearance of the aUCL was different in children than adults on MRI [14]. It is from these previous studies that the location of the aUCL becomes a clinical question regarding the need to know the origin of the ligament relative to the medial epicondyle apophysis for both potential reconstructions of that ligament and any deleterious effects that medial 
epicondyle fractures may have on the function of this important elbow stabilizer.

To our knowledge, there has been no previous study evaluating the relationship between the medial epicondyle apophysis and the origin of the aUCL in children throughout maturity. The purpose of this study was to determine the gender-specific anatomic origins of the aUCL in relation to the medial epicondyle apophysis based on different age groups of skeletally maturity.

\section{Materials and methods}

A retrospective review was performed on all elbow magnetic resonance imaging (MRI) obtained between September 2009 and April 2012 at our tertiary care children's hospital after institutional review board approval. Inclusion criteria for selecting the MRI studies included the following diagnoses: elbow pain, osteochondritis dissecans, and septic arthritis. Exclusion criteria included poor imaging quality, elbow flexion contracture, prior elbow injury or surgery obscuring the structures, a non-ossifying medial epicondyle, or a closed apophysis without an identifiable physeal scar. The subjects were grouped by gender and age groups based on relative skeletal maturity: the youngest group was 6-10 years old, the transitional group was 11-13 years old, and the older group was 14-18 years.

Standard MR imaging of the elbow at our institution was performed using either a GE 1.5-T Signa HDxt or Horizon 450 MRI scanner (General Electric Healthcare, Milwaukee, WI, USA) or a Phillips 1.5-T Intera scanner (Phillips Healthcare, Andover, MA, USA). All measurements were made directly from the digital MR images using the radiographic measuring software AMICAS PACS (AMICAS, Inc., Brighton, MA, USA). Both coronal and axial T1- and T2-weighted images were utilized to determine the best image to make measurements. They utilized 4.0-mm-thick cuts. Therefore, sagittal imaging was not utilized because these sequences were in the plane of the ligament, preventing accurate identification of the ligament given the available slice thickness. Repeat measurements were performed to obtain a mean final value by a single observer instructed and guided by a fellowship-trained musculoskeletal pediatric radiologist. The center of the insertion, the most prominent point on the sublime tubercle of the ulna, was identified on MR imaging. This point was used to identify the anterior bundle of the UCL, which was then traced proximally to its origin on the medial epicondyle (Fig. 2).

After confirming the criteria, data points were collected for each patient. The width of the aUCL at its origin was measured using the ruler tool from AMICAS. The measured distance from the center of the aUCL bundle on the medial epicondyle to the most lateral aspect of the

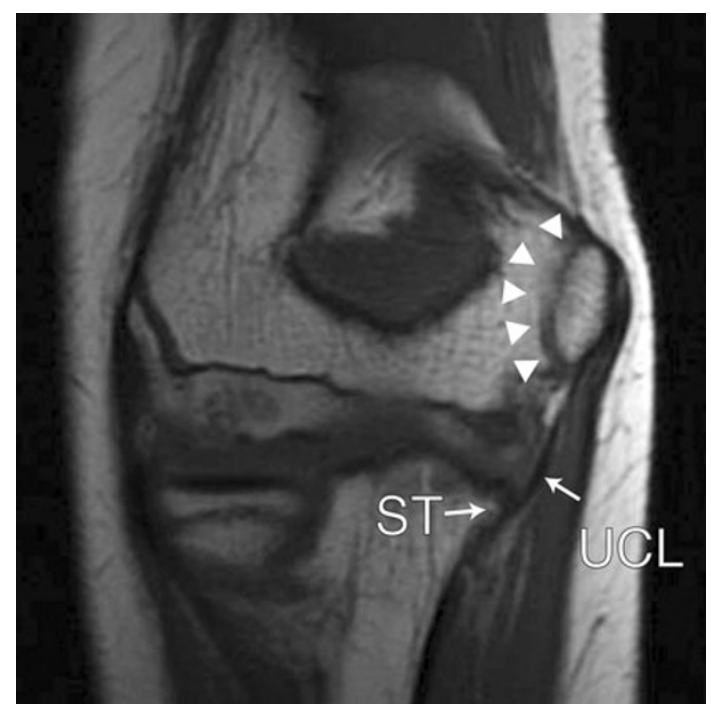

Fig. 2 Coronal T1 fat-saturated MRI image demonstrating the sublime tubercle of the ulna $(S T)$, the apophysis of the medial epicondyle (arrowheads), and the ulnar collateral ligament (UCL)

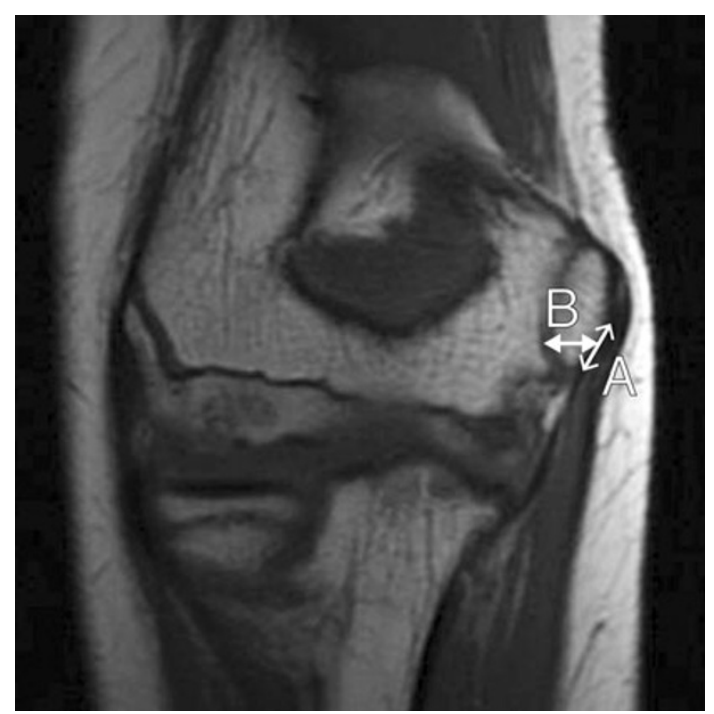

Fig. 3 Coronal T1 fat-saturated MRI images demonstrating the width of the UCL origin along the medial epicondyle $(A)$ and the distance from the medial epicondyle apophysis to the midpoint of the UCL origin $(B)$

cartilaginous portion of the apophysis on the axial images (which were set as the reference point for the relative measures of origin for the aUCL) were recorded. The distance from the midpoint of the aUCL origin to the reference point defined for the medial epicondyle apophysis was then recorded. A positive value was recorded for a distance that represented an aUCL midpoint lateral to the cartilaginous apophysis reference point (towards the center of the distal humerus), whereas a negative value was recorded for an origin that represented a medial midpoint (towards the secondary center of ossification) (Fig. 3). 
Statistical analysis

Means and statistical variations were then performed with significance set at $p<0.05$. The Kolmogorov-Smirnov test and Levene's test were performed to determine the normal distribution of the data, followed by two-tailed Pearson correlations for the width and midpoint of insertion for the entire group as well as the separate genders. SPSS v.12 (SPSS Inc., Chicago, IL, USA) was utilized for all statistical analyses.

\section{Results}

Ninety children ( 68 boys, 22 girls) with a mean age of 12.8 years (boys 13.3, girls 11.2) met the inclusion criteria. Kolmogorov-Smirnov tests and Levene's tests for ages and genders were all not significant. For the entire group of children, the mean aUCL width was $4.0 \mathrm{~mm}$ (range 2.8-5.8 $\mathrm{mm}$ ); and the apophysis to aUCL midpoint mean was $3.0 \mathrm{~mm}$ medial, with a maximum lateral position of $3.3 \mathrm{~mm}$ and a maximum medial position of $7.6 \mathrm{~mm}$ (SD $2.1 \mathrm{~mm}$ ). The entire group did not demonstrate a correlation with age in regards to width of aUCL $(r=0.264)$ or midpoint of aUCL to apophysis $(r=0.283)$.

Fifteen children were grouped into the young 6- to 10-year-old group (5 boys, 10 girls), 34 children were grouped in the transitional group ( 27 boys, 7 girls), and 41 adolescents were placed in the older than 14-year-old group ( 36 boys, 5 girls). All children had an intact aUCL by MRI assessment on the imaged elbow. First, the width of the UCL origin along the medial epicondyle was measured. Across all age groups, boys were found to have a wider UCL than girls $(4.05 \pm 0.16 \mathrm{~mm}$ vs $3.72 \pm 0.20 \mathrm{~mm}, p=0.03)$. Additionally, within each age group, the measured aUCL width in boys was greater than that in girls, although the differences were not statistically significant (Table 1). Among boys, the

Table 1 Table demonstrating average \pm standard deviation for UCL width (mm)

\begin{tabular}{llllll}
\hline & All ages & $<11$ & 11 to 13 & $>13$ & $p$ value \\
\hline Boys & $4.1 \pm 0.7$ & $3.8 \pm 0.4$ & $4.1 \pm 0.6$ & $4.1 \pm 0.7$ & $>0.05$ \\
Girls & $3.7 \pm 0.5$ & $3.7 \pm 0.5$ & $3.8 \pm 0.5$ & $3.6 \pm 0.5$ & $>0.05$ \\
$p$ value & 0.03 & 0.77 & 0.21 & 0.23 & \\
\hline
\end{tabular}

width of the aUCL increased from the youngest age group to the transitional group but plateaued into the older group. In contrast, the measured width of the aUCL in girls was fairly constant among the three age groups, but appeared slightly smaller in the older age group.

When comparing the entire cohort, there was no significant difference between the anatomical origin of the aUCL relative to that of the medial epicondyle apophysis $(p=0.52)$. Furthermore, within each age group, there was no difference in the anatomical origin of the aUCL between boys and girls (Table 2, $p>0.05$ ). When looking at genders independently, and comparing boys across age groups, there was also no significant difference (Table 2). However, when comparing girls across age groups, there was a trend between the youngest group and the oldest group, with the younger children exhibiting an aUCL midpoint that was more medial than the older patients, though in both groups the midpoint was medial to the apophyseal cartilage $(p=0.053)$.

\section{Discussion}

The biomechanics of the elbow results in the transfer of significant strain on the anterior band of the ulnar collateral ligament when the elbow undergoes valgus, as is seen during throwing or overhand swinging motions. Consequently, injury to this key stabilizer is not uncommon, and reports in the literature date back to 1946, when an injury to the ulnar collateral ligament was first described in a javelin thrower [9]. Since then, the injury and its reconstruction have been documented thoroughly in the literature. The conservative and operative management of UCL injuries in adults is well described. The surgical techniques for reconstructing this critical ligament have evolved, and more novel approaches continue to be explored. The success of these operations has improved significantly since the original report by Jobe and colleagues in 1986, with a recent systematic review of aUCL reconstruction reporting excellent outcomes, defined as the ability to return to the same or higher level of competition within 1 year, in $>80 \%$ of patients [15].

It is not clear, however, how these results translate to the pediatric population. Several factors, including the child's age, level of athletic achievement, access to quality

Table 2 Demonstrates the average \pm standard deviation for UCL midpoint distance to apophysis

\begin{tabular}{lllccc}
\hline & All ages & $<11$ & 11 to 13 & $>13$ & $p$ value \\
\hline Boys & $-3.1 \pm 2.3$ & $-2.5 \pm 0.9$ & $-3.5 \pm 12.5$ & $-2.9 \pm 2.2$ & $>0.05$ \\
Girls & $-2.8 \pm 1.5$ & $-3.1 \pm 2.3$ & $-3.11 \pm 2.3$ & $-3.11 \pm 2.3$ \\
$p$ value & 0.52 & 0.18 & 0.58 & 0.21 \\
\hline
\end{tabular}


rehabilitation, and the extent of the injury can be markedly different. The recent systematic review discussed above comprised studies of patients with mean ages of between 21 and 24 years old; a significant number of the subjects were professional athletes, ranging from 31 to $65 \%$ of the cohorts [10], with exceptional baseline fitness and unparalleled access to training and rehabilitation. Most previous studies have this bias away from the pediatric population [2]. Additionally, UCL injuries in children tend to be more acute than those in adults, resulting in a different quality of ligamentous injury [1]. These factors make it difficult to anticipate outcomes in the pediatric population. More important may be the implication of elbow instability seen with medial epicondyle fractures, which is much more common in the pediatric age group [7]. Our data clearly demonstrate that the aUCL is attached to the apophysis of the medial epicondyle in both genders and at all levels of skeletal immaturity, highlighting the potential function deficit or loss of stability that could be seen with an indirect injury to the ligament via fracture.

The treatment considerations in younger athletes are also different from those for their older counterparts. Younger athletes have greater healing potential than their older counterparts; thus, conservative treatments may be more effective but come at the cost of lengthy absences from the sport and a lost opportunity to develop athletically. Additionally, many patients would recover without surgery and could participate in a more restricted athletic role that reduces elbow stress, but this may be seen as an unfavorable outcome to the athlete. Finally, given the yearlong recovery process and the relative success of conservative treatments, the decision to operate should be considered with the child's talent and career prospects in mind-an assessment that may be both difficult and unpopular [6].

Consequently, surgical indications and guidelines for aUCL reconstruction in the pediatric and adolescent population are different from those in the collegiate or professional athlete. In addition to the factors discussed above, surgical reconstruction of the aUCL in the pediatric population requires consideration of the developing anatomy of the elbow. Specifically, the surgeon must choose the origin of the reconstructed ligament with respect to the medial epicondyle apophysis, a relationship that was nebulous prior to the completion of this present study. Our results demonstrate that the anatomic origin of the aUCL is relatively consistent across age and gender for children with an open medial epicondyle apophysis centered approximately $3 \mathrm{~mm}$ medial to the lateral edge of the apophysis. From a surgical perspective, reconstruction of this ligament in this age group places the apophysis at risk for injury and possible partial arrest. Even if a relatively small graft is used with careful juxtaposition of the tunnel in a non-anatomic lateralized position, there is a potential risk of injury to the apophyseal cartilage from heat generated during the drilling process. However, an anatomically placed tunnel within the apophysis clearly risks the growth of the medial epicondyle. The clinical significance of a complete or partial apophyseal arrest at the medial epicondyle is unknown.

The most significant limitation on this study was that all the MR images utilized were from children with pain or pathology of the elbow. A prospective study of normal elbows in children would have eliminated this potential confounding factor. Another limitation is that there were relatively small numbers in the youngest group and in all the female gender groups. However, when we performed a power analysis of this study and assessed the effect size (for reference: large effect, $\eta=0.4$; moderate effect, $\eta=0.25$; low effect, $\eta=0.1$, we found the following in regards to these two populations in our cohort. A large effect size was observed when comparing the youngest group of boys to girls in aUCL midpoints $(\eta=0.37)$, with a subsequent power analysis indicating that a total sample of 60 patients was needed in this young age group to reach $80 \%$ power. Similarly, a large effect for the female gender comparison of aUCL midpoint across the age groups was observed $(\eta=0.46)$, with a power analysis revealing that approximately 50 girls were needed to reach $80 \%$ power. In other words, although this study is underpowered, the size of the effect observed does indicate for both of these comparisons that there is likely a difference. In order to achieve the same effect size and have power, it would be necessary to perform a prospective study on over 1,200 children with normal elbows. This study appears to provide results that support (with large effect sizes) the hypothesis that the UCL is larger in boys, and in all cases originates within the apophyseal cartilage of the medial epicondyle. However, the clinical significance of the gender difference is minimal relative to the significance that the origin of the aUCL is always medial to the lateral aspect of the apophyseal cartilage of the medial epicondyle.

Increasingly, youth athletes are engaging in overuse throwing habits, resulting in substantial and repeated microtrauma to the developing throwing elbow. Often, there is inadequate time for healing, causing a cumulative injury that weakens the UCL and renders the tissue prone to failure. While the clinical results of UCL reconstruction in young athletes are roughly equal to those of older athletes [16], there are important factors that warrant complex consideration by a surgeon contemplating such an operation. Should a surgical reconstruction be indicated, operative techniques should be chosen in consideration of it being a pediatric elbow and the risk it poses to the development of the elbow. However, our study suggests that the origin for the reconstructed ligament in relation to the 
medial epicondyle apophysis does not require specific consideration of either age or gender.

Conflict of interest The authors declare that they have no conflict of interest.

\section{References}

1. Greiwe RM, Saifi C, Ahmad CS (2010) Pediatric sports elbow injuries. Clin Sports Med 29:677-703

2. Cain EL Jr, Andrews JR, Dugas JR, Wilk KE, McMichael CS, Walter JC 2nd, Riley RS, Arthur ST (2010) Outcome of ulnar collateral ligament reconstruction of the elbow in 1,281 athletes: results in 743 athletes with minimum 2 year follow-up. Am J Sports Med 38:2426-2434

3. Dugas JR, Ostrander RV, Cain EL, Kingsley D, Andrews JR (2007) Anatomy of the anterior bundle of the ulnar collateral ligament. J Shoulder Elbow Surg 16:657-660

4. Fleisig GS, Andrews JR (2012) Prevention of elbow injuries in youth baseball pitchers. Sports Health 4:419-424

5. Kramer DE (2010) Elbow pain and injury in young athletes. J Pediatr Orthop 30:S7-S12

6. Petty DH, Andrews JR, Fleisig GS, Cain EL (2004) Ulnar collateral ligament reconstruction in high school baseball players: clinical results and injury risk factors. Am J Sports Med 32:1158-1164

7. Woods GW, Tullos HS (1977) Elbow instability and medial epicondyle fractures. Am J Sports Med 5:23-30
8. Schwab GH, Bennett JB, Woods GW, Tullos HS (1980) Biomechanics of elbow instability: the role of the medial collateral ligament. Clin Orthop Relat Res 146:42-52

9. Rettig AC, Sherrill C, Snead DS, Mendler JC, Mieling P (2001) Nonoperative treatment of ulnar collateral ligament injuries in throwing athletes. Am J Sports Med 29:15-17

10. Vitale MA, Ahmad CS (2008) The outcome of elbow ulnar collateral ligament reconstruction in overhead athletes: a systematic review. Am J Sports Med 36:1193-1205

11. Olsen SJ 2nd, Fleisig GS, Dun S, Loftice J, Andrews JR (2006) Risk factors for shoulder and elbow injuries in adolescent baseball pitchers. Am J Sports Med 34:905-912

12. Savoie FH 3rd, Trenhaile SW, Roberts J, Field LD, Ramsey JR (2008) Primary repair of ulnar collateral ligament injuries of the elbow in young athletes: a case series of injuries to the proximal and distal ends of the ligament. Am J Sports Med 36:1066-1072

13. Wei AS, Khana S, Limpisvasti O, Crues J, Podesta L, Yocum LA (2010) Clinical and magnetic resonance imaging findings associated with little league elbow. J Pediatr Orthop 30:715-719

14. Sugimoto H, Ohsawa T (1994) Ulnar collateral ligament in the growing elbow: MR imaging of normal development and throwing injuries. Radiology 192:417-422

15. Purcell DB, Matava MJ, Wright RW (2007) Ulnar collateral ligament reconstruction: a systematic review. Clin Orthop Relat Res 455:72-77

16. Azar FM, Andrews JR, Wilk KE, Groh D (2000) Operative treatment of ulnar collateral ligament injuries of the elbow in athletes. Am J Sports Med 28:16-23 